\title{
Bose-Einstein Condensation and Quasicrystals
}

\author{
M. Alexanian ${ }^{1}$, V.E. Mkrtchian ${ }^{2}$ \\ ${ }^{1}$ Department of Physics and Physical Oceanography, \\ University of North Carolina Wilmington, Wilmington, NC 28403-5606, USA \\ ${ }^{2}$ Institute for Physical Research, Armenian Academy of Sciences, Ashtarak 0203, Republic of Armenia
}

E-mail: alexanian@uncw.edu

Received 22 June 2021

\begin{abstract}
We consider interacting Bose particles in an external local potential. It is shown that large class of external quasicrystal potentials cannot sustain any type of Bose-Einstein condensates. Accordingly, at spatial dimensions $D \leq 2$ in such quasicrystal potentials a supersolid is not possible via Bose-Einstein condensates at finite temperatures. The latter also hold true for the two-dimensional Fibonacci tiling. However, supersolids do arise at $D \leq 2$ via Bose-Einstein condensates from infinitely long-range, nonlocal interparticle potentials.
\end{abstract}

https://doi.org/10.52853/18291171-2021.14.2-128

\section{Introduction}

In a recent paper [1], the question of the existence of a Bose-Einstein condensate (BEC) in a supersolid was investigated. It was shown that an external crystalline lattice potential could not by itself sustain a condensate and so a crystalline lattice potential cannot give rise to a supersolid via a BEC. In addition, it was found that for spatial dimensions $D \leq 2$ self-crystallization occurs if the interparticle interaction between bosons is nonlocal and of infinitely long-range. In what following, we consider the same issues but now addressing quasicrystals, as well as, the 2-dimensional square Fibonacci tiling, which does not posses one of the "forbidden" $n$-fold rotational symmetries, $n \geq 5$, that are characteristic of quasicrystals and incompatible with translational periodicity.

\section{Crystals}

The Hamiltonian for the interacting Bose gas is

$$
\begin{aligned}
\hat{H} & =\int d \mathbf{r} \hat{\psi}^{\dagger}(\mathbf{r})\left(\frac{-\hbar^{2}}{2 m} \nabla^{2}\right) \hat{\psi}(\mathbf{r})+\int d \mathbf{r} \hat{\psi}^{\dagger}(\mathbf{r}) V_{e x t}(\mathbf{r}) \hat{\psi}(\mathbf{r}) \\
& +\int d \mathbf{r}_{1} \int d \mathbf{r}_{2} \int d \mathbf{r}_{3} \int d \mathbf{r}_{4} \hat{\psi}^{\dagger}\left(\mathbf{r}_{1}\right) \hat{\psi}^{\dagger}\left(\mathbf{r}_{2}\right) V\left(\mathbf{r}_{1}, \mathbf{r}_{2}, \mathbf{r}_{3}, \mathbf{r}_{4}\right) \hat{\psi}\left(\mathbf{r}_{4}\right) \hat{\psi}\left(\mathbf{r}_{3}\right),
\end{aligned}
$$

where $V_{\text {ext }}(\mathbf{r})$ is an arbitrary, external potential, $V\left(\mathbf{r}_{1} ; \mathbf{r}_{2} ; \mathbf{r}_{3} ; \mathbf{r}_{4}\right)$ is a general two-particle interaction potential, and $\hat{\psi}(\mathbf{r})$ and $\hat{\psi}^{\dagger}(\mathbf{r})$ are bosonic field operators that destroy or create a particle at spatial position $\mathbf{r}$, respectively.

Macroscopic occupation in the single-particle state $\psi(\mathbf{r})$ result in the non-vanishing [2] of the quasi-average $\psi(\mathbf{r})=<\hat{\psi}(\mathbf{r})>$ and so the boson field operator

$$
\hat{\psi}(\mathrm{r})=\psi(\mathrm{r})+\hat{\varphi}(\mathrm{r}),
$$


where

$$
\hat{\varphi}(\mathrm{r})=\sqrt{\frac{1}{V(D)}} \sum_{\mathbf{k}} \hat{a}_{\mathbf{k}} \mathrm{e}^{\mathbf{k} \cdot \mathbf{r}}
$$

with the condensate wavefunction

$$
\psi(\mathbf{r})=\sqrt{\frac{N_{0}}{V(D)}} \sum_{\mathbf{k}^{\prime}} \xi_{\mathbf{k}^{\prime}} e^{i \mathbf{k}^{\prime} \cdot \mathbf{r}},
$$

and normalization

$$
\sum_{\mathbf{k}^{\prime}}\left|\xi_{\mathbf{k}^{\prime}}\right|^{2}=1,
$$

where $N_{\boldsymbol{o}}$ is the number of atoms in the condensate, $V(\mathrm{D})$ is the D-dimensional "volume," $\hat{a}_{\mathbf{k}}^{\dagger}\left(\hat{a}_{\mathbf{k}}\right)$ are the creation (annihilation) operators with commutation relations $\left[\hat{a}_{\mathbf{k}}, \hat{a}_{\mathbf{k}^{\prime}}^{\dagger}\right]=\delta_{\mathbf{k}, \mathbf{k}^{\prime}}$, and $<\hat{\varphi}(\mathbf{r})>=0$. The operator $\hat{\varphi}(\mathbf{r})$ has no Fourier components with momenta $\left\{\mathbf{k}^{\prime}\right\}$ that are macroscopically occupied and so $\int d \mathbf{r} \hat{\varphi}^{\dagger}(\mathbf{r}) \psi(\mathbf{r})=0$. The separation of $\hat{\psi}(\mathbf{r})$ into two parts gives rise to the following (gauge invariance) symmetry breaking term [2] associated with the interparticle potential in the Hamiltonian (1)

$$
\begin{aligned}
\hat{H}_{\text {symm }} & =\int d \mathbf{r}_{1} \hat{\varphi}^{\dagger}\left(\mathbf{r}_{1}\right) \int d \mathbf{r}_{2} \int d \mathbf{r}_{3} \int d \mathbf{r}_{4} \psi^{*}\left(\mathbf{r}_{2}\right)\left[V\left(\mathbf{r}_{1}, \mathbf{r}_{2}, \mathbf{r}_{3}, \mathbf{r}_{4}\right)+V\left(\mathbf{r}_{2}, \mathbf{r}_{1}, \mathbf{r}_{3}, \mathbf{r}_{4}\right)\right] \psi\left(\mathbf{r}_{3}\right) \psi\left(\mathbf{r}_{4}\right)+\text { h.c. } \\
& \equiv \int d \mathbf{r}_{1} \hat{\varphi}^{\dagger}\left(\mathbf{r}_{1}\right) \chi\left(\mathbf{r}_{1}\right)+\text { h.c. }
\end{aligned}
$$

Recall that the interaction potential between bosons indicates that macroscopic occupation in a single-particle linear momentum state, viz., a spatially uniform condensate, does not give rise to additional macroscopic occupation in any other single-particle linear momentum states owing to the conservation of linear momentum by the interaction [1]. However, macroscopic occupation in two or more single-particle linear momentum states give rise to a denumerably infinite, macroscopically occupied states. For instance, macroscopic occupation in the single-particle momenta states $\mathbf{k}$, $\mathbf{k} \pm \mathbf{q}_{1}$, and $\mathbf{k} \pm \mathbf{q}_{2}$, where $\mathbf{q}_{1} \times \mathbf{q}_{2} \neq \mathbf{0}$, gives rise to additional macroscopic occupation in the singleparticle momenta states $\mathbf{k}+n_{1} \mathbf{q}_{1}+n_{2} \mathbf{q}_{2}$, with $n_{1}, n_{2}=0, \pm 1, \pm 2, \cdots \cdots$ owing to the symmetry breaking term $\hat{H}_{\text {symm }}$.

Accordingly, the condensate wave function gets augmented and is of the Block form given by

$$
\begin{aligned}
\psi_{\mathbf{k}}(\mathbf{r}) & =\sqrt{\frac{N_{0}}{V(D)}} \sum_{n_{1}, n_{2}=-\infty}^{\infty} \xi_{\mathbf{k}+n_{1} \mathbf{q}_{1}+n_{2} \mathbf{q}_{2}} e^{i\left(\mathbf{k}+n_{1} \mathbf{q}_{1}+n_{2} \mathbf{q}_{2}\right) \cdot \mathbf{r}} \\
& \equiv e^{i \mathbf{k} \cdot \mathbf{r}} u_{\mathbf{k}}(\mathbf{r}),
\end{aligned}
$$

with $u \mathbf{k}\left(\mathbf{r}+\mathbf{r}_{\mathbf{0}}\right)=u \mathbf{k}(\mathbf{r})$, where

$$
\mathbf{r}_{0}=2 \pi\left[\frac{\left(q_{2}^{2}-\mathbf{q}_{1} \cdot \mathbf{q}_{2}\right) \mathbf{q}_{1}+\left(q_{1}^{2}-\mathbf{q}_{1} \cdot \mathbf{q}_{2}\right) \mathbf{q}_{2}}{q_{1}^{2} q_{2}^{2}-\left(\mathbf{q}_{1} \cdot \mathbf{q}_{2}\right)^{2}}\right]
$$

\section{Quasicrystals}

We now consider the replacement (2) in the term in (1) associated with the external, local potential $V_{\text {ext }}(\mathbf{r})$. One obtains the symmetry breaking Hamiltonian 


$$
\hat{H}_{\text {symm }}^{(e x t)}=\int \mathrm{d} \mathbf{r} \hat{\varphi}^{\dagger}(\mathbf{r}) V_{\text {ext }}(\mathbf{r}) \psi(\mathbf{r})+\text { h.c. }
$$

Consider the local, finite two-dimensional quasicrystal lattice potential,

$$
V_{\text {ext }}(\mathbf{r})=\frac{1}{(2 \pi)^{2}} \sum_{\mathbf{k}} g(\mathbf{k}) \sum_{m_{1} \cdots m_{n}=-M_{1} \cdots-M_{n}}^{M_{1} \cdots M_{n}} e^{-i \mathbf{k} \cdot\left(\mathbf{r}-\sum_{i=1}^{n} m_{i}\left(\alpha_{i} \mathbf{a}+\beta_{i} \mathbf{b}\right)\right)}
$$

where $g(\mathbf{k})$ is the Fourier transform, $\mathbf{a}$ and $\mathbf{b}$ are arbitrary two-dimensional vectors in the $\mathrm{x}$ $\mathrm{y}$ plane with $\mathbf{a} \times \mathbf{b} \neq 0,\left(\alpha_{i} \mathbf{a}+\beta_{i} \mathbf{b}\right) \times\left(\alpha_{j} \mathbf{a}+\beta_{j} \mathbf{b}\right) \neq 0, \alpha_{i}$ and $\beta_{i}$ are irrational numbers, and $n \geq 3$. In (10), we have projected a periodic structure in $n$-dimensional space into a $D$-dimensional quasicrystal space $(n>D)$. Cases $n=1,2$ reduce to a one- and twodimensional crystals, respectively. One obtains that

$$
\begin{aligned}
\hat{H}_{\text {symm }}^{(e x t)} & =\int \mathrm{d} \mathbf{r} \hat{\varphi}^{\dagger}(\mathbf{r}) V_{\text {ext }}(\mathbf{r}) \psi(\mathbf{r})+\text { h.c. } \\
& =\frac{\sqrt{N_{0}}}{V(D)} \sum_{\mathbf{k}_{1}, \mathbf{k}_{2} \neq \mathbf{k}_{1}} \hat{a}_{\mathbf{k}_{1}}^{\dagger} \xi_{\mathbf{k}_{2}} g(\mathbf{k}) \prod_{i=1}^{n} \frac{\sin \left[\mathbf{k} \cdot\left(\alpha_{i} \mathbf{a}+\beta_{i} \mathbf{b}\right)\left(M_{i}+1 / 2\right)\right]}{\sin \left[\mathbf{k} \cdot\left(\alpha_{i} \mathbf{a}+\beta_{i} \mathbf{b}\right) / 2\right]}+h . c .,
\end{aligned}
$$

where $\mathbf{k} \equiv \mathbf{k}_{2}-\mathbf{k}_{1}$, which follows with the aid of (3), (4), and (10). Recall that

$$
\sum_{m=-M}^{M} e^{i m x}=\frac{\sin [x(M+1 / 2)]}{\sin [x / 2]} \rightarrow 2 \pi \delta(x) \quad(M \rightarrow \infty) .
$$

Note that $\mathbf{k}_{\mathbf{1}} \neq \mathbf{k}_{\mathbf{2}}$, that is, $\mathbf{k} \neq \mathbf{0}$, since $\mathbf{k}_{\mathbf{2}}$ is in the condensate and $\mathbf{k}_{\mathbf{1}}$ is not in the condensate. Therefore, $\hat{H}_{\text {symm }}^{(e x t)}$ vanishes for arbitrary BEC in the macroscopically large aperiodic lattice limit whichever order the limits are taken. Therefore, one cannot generate a two-dimensional supersolid via a BEC at temperatures $T \geq 0$ from an external aperiodic lattice potential. However, a twodimensional supersolid at finite temperatures can be generated via long- range, nonlocal potentials provided by the interparticle interaction which results in self-organization [1], much as Wigner crystallization or Wigner lattice, electrons moving in a uniform background of positive charge that restore electric neutrality [3].

The embedded spaces of D-dimensional quasiperiodic structures are abstract spaces whose dimensions are more than three. The dimensions of the embedded space are dependent on the symmetry of the quasicrystal $(D>1)[4,5]$. For example, the quasicrystals with $5,8-, 10-$, and $12-$ fold symmetry need to be embedded into four-dimensional space, $n=4$. While for the quasiperiodic structures with 7-, 9-, 18-fold symmetry, the dimension of the embedding spaces increases [4-6] to six, $n=6$.

The Fibonacci tiling [7,8] does not fall in the above class of lattice potentials given by (10). However, the Fourier transform of the Fibonacci sequence has $\delta$-function peaks at $k=2 \pi\left(m+m^{\prime} \tau\right) / \sqrt{5}$, where $\boldsymbol{\tau}=(1+\sqrt{5}) / 2$ is the golden mean and $m$ and $m^{\prime}$ are integers [9]. Expressed in terms of Fourier transforms (9) becomes

$$
\hat{H}_{s y m m}^{(e x t)}=\frac{\sqrt{N_{0}}}{V(D)} \sum_{\mathbf{k}^{\prime} \mathbf{k}} \hat{a}_{\mathbf{k}}^{\dagger} \xi_{\mathbf{k}^{\prime}} \tilde{V}_{e x t}\left(\mathbf{k}^{\prime}-\mathbf{k}\right)+h . c .
$$

where

$$
\tilde{V}_{e x t}\left(\mathbf{k}^{\prime}-\mathbf{k}\right)=\int \mathrm{d} \mathbf{r} V_{e x t}(\mathbf{r}) e^{i\left(\mathbf{k}^{\prime}-\mathbf{k}\right) \cdot \mathbf{r}} .
$$

Consider the case where $\tilde{V}_{\boldsymbol{e x t}}\left(\mathbf{k}^{\prime}-\mathbf{k}\right)$ is given by a sum of Dirac $\delta$-functions, which is the case for the Fibonacci tiling [9]. Now the vector $\mathbf{k}^{\prime}-\mathbf{k}$ must lie either in the condensate or outside the condensate. In either case, $\hat{H}_{s y m m}^{(e x t)}$ vanishes for arbitrary BEC since the vector $\boldsymbol{k}$ is not in the condensate while the vector $\mathbf{k}^{\prime}$ is in the condensate. 


\section{Quasicrystal condensate}

The necessity that a BEC has the Bloch form and represents a self-organized supersolid for $D \leq 2$ requires that the interaction between the atoms be nonlocal and of infinitely long-range [10]. This proof also applies for the existence of an aperiodic condensate. For instance, macroscopic occupation in the single-particle momenta states $\mathbf{0}, \mathbf{q}_{1}, \alpha_{1} \mathbf{q}_{1}, \mathbf{q}_{2}$, and $\alpha_{2} \mathbf{q}_{2}$, where $\alpha_{1}$ and $\alpha_{2}$ are irrational numbers and $\mathbf{q}_{1} \times \mathbf{q}_{2} \neq \mathbf{0}$, gives rise to additional macroscopic occupation in the singleparticle momenta states $\left(m_{1}+\alpha_{1} m_{2}\right) \mathbf{q}_{1}+\left(n_{1}+\alpha_{2} n_{2}\right) \mathbf{q}_{2}$, with $m_{1}, m_{2}, n_{1}, n_{2}=0, \pm 1, \pm 2, \cdots$ owing to the symmetry breaking term $\hat{H}_{\text {symm }}$ and the linear momentum conservation of the interparticle potential.

Accordingly, the condensate wave function gets augmented and is given by

$$
\psi(\mathbf{r})=\sqrt{\frac{N_{0}}{V(D)}} \sum_{m_{1}, m_{2}, n_{1}, n_{2}=-\infty}^{\infty} \xi_{\left(m_{1}+\alpha_{1} m_{2}\right) \mathbf{q}_{1}+\left(n_{1}+\alpha_{2} n_{2}\right) \mathbf{q}_{2}} e^{i\left[\left(m_{1}+\alpha_{1} m_{2}\right) \mathbf{q}_{1}+\left(n_{1}+\alpha_{2} n_{2}\right) \mathbf{q}_{2}\right] \cdot \mathbf{r}},
$$

where $\mathbf{q}_{1}$ and $\mathbf{q}_{2}$ are crystallographic directions.

\section{Summary and Discussion}

We have established that supersolids in $D \leq 2$ cannot be generated via Bose-Einstein condensates in a wide class of quasicrystal potentials that includes the Fibonacci tiling. However, supersolids do arise via Bose-Einstein condensates from infinitely long-range, nonlocal interparticle potentials.

\section{References}

[1] M. Alexanian, V.E. Mkrtchian, Arm J. Phys. 14 (2021) 29.

[2] N.N. Bogoliubov, Physica 26 (1960) S1.

[3] E. Wigner, Phys. Rev. 46 (1934) 1002.

[4] W. Steurer, S. Deloudi, Crystallography of Quasicrystals: Concepts, Methods and Structures (Springer-Verlag, 2009).

[5] H. Heller, Acta Crystallogr. A, Found. Crystallogr. 41 (1985) 541.

[6] T. Janssen, G. Chapuis, M. De Boissieu, Aperiodic Crystals: From Modulated Phases to Quasicrystals (Oxford University Press, USA, 2007).

[7] R. Lifshitz, J. Alloys Compd. 342 (2002) 186.

[8] A. Jagannathan, The Fibonacci quasicrystal: case study of hidden dimensions and multifractality, arXiv:2012.14744 (2020).

[9] D. Levine, P.J. Steinhardt, Phys. Rev. Lett. 53 (1984) 2477.

[10] M. Alexanian, Arm J. Phys. 11 (2018) 117. 